\title{
ON FUNCTIONS SATISFYING THE MEAN VALUE PROPERTY WITH RESPECT TO A PRODUCT MEASURE
}

\author{
A. M. GARSIA AND E. RODEMICH
}

Let $\mu(y)$ denote a Borel-Stieltjes measure on the $d$-dimensional coordinate space $E_{d}$ and let its support $S$ be compact. Assume further that

$$
\int_{S} d \mu(y)=1
$$

A function $u(x)=u\left(x_{1}, x_{2}, \cdots, x_{d}\right)$ defined and, to avoid technicalities, continuous in a region $D$ of $E_{d}$ is said to satisfy the mean value property with respect to $\mu(y)$ if for all $x \in D$ we have

$$
u(x)=\int_{S} u(x+t y) d \mu(y)
$$

for all sufficiently small $t$. For example, the characteristic feature of the ordinary harmonic functions is that they satisfy the mean value property with respect to the homogeneous distribution on the unit sphere.

It has been shown [4], [6] that functions satisfying a mean value property are harmonic in some sense; however, unless the given mass is of a very special nature it is difficult even to decide whether or not the space of functions satisfying the mean value property with respect to it is infinite dimensional. Results in this topic are to be found in $[1],[2],[3],[4],[5],[6],[7]$ and $[8]$.

Our main result here is a proof that whenever $\mu(y)$ is a nondegenerate product mass the space of functions satisfying the mean value property with respect to $\mu(y)$ is necessarily finite dimensional. "Nondegenerate" here is to mean that the support of the measure is not contained in a hyperplane through the origin. In particular as a corollary we obtain that the spaces of functions satisfying the mean value property with respect to homogeneous distribution on squares, rectangles and more generally on $d$-dimensional parallelepipedons are finite dimensional. The latter results are new for $d \geqq 3$.

The starting point in establishing these facts is the following result established by A. Friedman and W. Littman [6] and indirectly also by L. Flatto [4].

Basic criterion. The space of functions satisfying the mean vaiue

Received by the editors October 22, 1965. 
property with respect to $\mu(y)$ is finite dimensional if and only if the polynomials

(2) $P_{n}\left(\xi_{1}, \xi_{2}, \cdots, \xi_{d}\right)=\int_{S}\left(\xi_{1} y_{1}+\xi_{2} y_{2}+\cdots+\xi_{d} y_{d}\right)^{n} d \mu(y) \quad(n \geqq 1)$

have no root in common other than $(0,0, \cdots, 0)$.

The condition expressed by this criterion can be transformed into a more convenient one. If we multiply the $n$th polynomial in (2) by $(i t)^{n} / n$ !, where $t$ is a complex parameter, and sum from 0 to $\infty$ we obtain the expression

$$
\int_{S} e^{i\left(\xi_{1} y_{1}+\xi_{2} y_{2}+\cdots+\xi_{d} y_{d}\right) t} d \mu(y)
$$

which is none other than the Fourier transform

$$
\Lambda\left(u_{1}, u_{2}, \cdots, u_{d}\right)=\int_{S} e^{i\left(u_{1} y_{1}+u_{2} y_{2}+\cdots+u_{d} y_{d}\right)} d \mu(y)
$$

of the measure $\mu(y)$ evaluated at the point

$$
\left(u_{1}, u_{2}, \cdots, u_{d}\right)=\left(t \xi_{1}, t \xi_{2}, \cdots, t \xi_{d}\right) .
$$

It is easy to see that the basic criterion can be reformulated as follows:

"The space of functions satisfying the mean value property with respect to $\mu(y)$ is infinite dimensional if and only if for some complex vector $\left(\xi_{1}, \xi_{2}, \cdots, \xi_{d}\right) \neq(0,0, \cdots, 0)$ we have

$$
\Lambda\left(t \xi_{1}, t \xi_{2}, \cdots, t \xi_{d}\right) \equiv 1
$$

for all complex $t . " 1$

We are now in a position to establish our result. In fact, suppose $\mu(y)$ is the product of $\mu_{1}\left(y_{1}\right), \mu_{2}\left(y_{2}\right), \cdots, \mu_{d}\left(y_{d}\right)$ and set for convenience

$$
\Lambda_{j}\left(u_{j}\right)=\int_{-\infty}^{+\infty} e^{i u_{j} y_{j}} d \mu_{j}\left(y_{j}\right) .
$$

Assume, if possible, that there is a nontrivial vector $\left(\xi_{1}, \xi_{2}, \cdots, \xi_{d}\right)$ for which (3) holds and let $J=\left\{j: \xi_{j} \neq 0\right\}$. From (3) we then get

$$
1 \equiv \prod_{j \in J} \Lambda_{j}\left(t \xi_{j}\right) \forall t
$$

1 This formulation, which was known to us since April, 1961, was independently noticed also by L. Flatto [5]. 
We thus conclude that none of the functions $\Lambda_{j}(u)$ for $j \in J$ ever vanishes. From the compactness of the mass $\mu(y)$ we deduce that $\Lambda_{j}(u)$ is entire of order one. Thus from the Hadamard factorization theorem it follows that for $j \in J$

$$
\Lambda_{j}(u)=e^{A_{j} u+B_{i}} .
$$

On the other hand by the very definition of these functions each $A_{j}$ is pure imaginary and each $B_{i}$ may be taken equal to one. We can thus write

$$
\Lambda_{j}(u)=e^{i a_{j} u} \quad j \in J .
$$

Substituting back in (4) we obtain

$$
\sum_{j \in J} a_{j} \xi_{j}=0
$$

Note then that (5) means that $\mu_{j}\left(y_{j}\right)$ is a unit mass concentrated at the point $y_{j}=a_{j}$. Hence equation (5) upon taking real (or imaginary) parts gives an equation

$$
\sum_{j \in J} y_{j} \operatorname{Re} \xi_{j}=0 \quad\left(\text { or } \sum_{j \in J} y_{j} \operatorname{Im} \xi_{j}=0\right)
$$

satisfied by every point of the support of $\mu(y)$. In other words $\mu(y)$ must be a degenerate measure.

\section{REFERENCES}

1. E. F. Beckenbach and M. Reade, Mean values and harmonic polynomials, Trans. Amer. Math. Soc. 53 (1943), 230-238.

2. - Regular solids and harmonic polynomials, Duke Math. J. 12 (1945), 629-644.

3. W. Brödel, Funktionen mit Gaussischer Mitteliwerteigenschaft für Konvexe Kurven und Bereiche, Deutsche Math. 4 (1939), 3-15.

4. L. Flatto, Functions with a mean value property, J. Math. Mech. 10 (1961), $11-18$.

5. - Functions with a mean value property. II, Amer J. Math. 5 (1963), 248270.

6. A. Friedman and W. Littman, Functions satisfying the mean value property, Trans. Amer. Math. Soc. 102 (1962), 167-180.

7. - Mean values and harmonic polynomials, Mineographed notes, Inst. of Tech., Univ. of Minnesota, Minneapolis, 1961.

8. A. Garsia, A note on the mean value property, Trans. Amer. Math. Soc. 102 (1962), 181-186.

California Institute of Technology, Pasadena 